\title{
Neoneurogenesis in squamous cell carcinoma of tongue: a promoter to its progression and metastasis
}

Zhenq Xu ( $\sim$ hugoxuarticles@hotmail.com)

Tianjin Stomatological Hospital https://orcid.org/0000-0001-5254-6724

\section{Jianw Shang}

Tianjin Stomatological Hospital

\section{Xu Xiang}

Tianjin Stomatological Hospital

Li Long

Tianjin Stomatological Hospital

\section{Yingb Yan}

Tianjin Stomatological Hospital

Jun Zhang

Tianjin Stomatological Hospital

\section{Research article}

Keywords: neoneurogenesis, squamous cell carcinoma of tongue, promoter, progression, metastasis

Posted Date: February 27th, 2020

DOI: https://doi.org/10.21203/rs.2.20272/v2

License: @ (1) This work is licensed under a Creative Commons Attribution 4.0 International License. Read Full License 


\section{Abstract}

Background: To explore whether neoneurogenesis is a predisposing factor to the development and metastasis of squamous cell carcinoma of tongue (TSCC) . Methods: Twenty patients diagnosed with TSCC were included. The objectives were divided into 4 groups according to WHO T-stage standard (five cases in each group) and 2 groups based on lymph node metastasis or not. Detection of neoneurogenesis in paraffin sections from primary tongue cancer tissues was stained by Neurofilament-Light chain antibody (NF-L) using immunohistochemistry method (IHC). The number of new growth nerve fiber bundles was calculated in each randomized view from 3 non-overlapping microscope fields of each tumor section under a light microscope at X 200. Differences in the density of nerve fiber bundles among the T-stage groups and lymph node metastasis groups were calculated using Chi-square test. An Q level of 0.05 was considered statistically significant. Results: The age of patients varied from 33 to 74 years with a mean age of $56.10 \pm 11.18$ years. Males were affected predominantly with a ratio of 1.5:1 to females. IHC staining of NF-L was positive in all 20 paraffin sections of TSCC which suggested that newly growth nerves (namely neoneurogenesis) were observed in tumor mciroenvironment. The intensity of newly formed nerve bundles increased with $T$ stage, and the difference was statistically significant between early stage (T1 $10 T 2)$ and late stage $(T 30 T 4)(p=0.000)$. Increased prevalence of newly growth nerve bundles correlated significantly to the cervical lymph node metastasis $(p=0.000)$. Conclusions: Neoneurogenesis in tumor microenviroment of TSCC is a predisposing factor to its aggressive development and cervical lymph node metastasis. From a therapeutic perspective, further studies on the topic may provide a new clinical opportunity through anti-neurogenesis.

\section{Background}

Squamous cell carcinoma of tongue (TSCC) is the most common reported malignant tumor in the oral cavity. TSCC is more aggressive than other cancers of the oral cavity because of the propensity for rapid local invasion and nodal or even distant spread. Despite significant advances have been made in its prevention, diagnosis, and treatment strategies in the last three decades, there has been only modest progress in the improvement of its prognosis mainly because of regional recurrence and cervical lymph node metastasis [1].

The exact pathogenesis of TSCC has not been uncovered till date. Tumor microenvironment (TME) of TSCC plays a crucial role in its origination and progression, in which new growth of blood vessels, namely "tumor angiogenesis" can transport blood supply, nutrient and oxygen to the tumors and stimulate the proliferation of cancer cells, while new formation of lymph vessels, namely "tumor lymphogenesis" actively contributes to cancer dissemination via the newly formed lymphatic vessels $[2,3]$.

Nerves are also a common feature of the TME, despite it is well demonstrated that nerve fibers are often passively invaded by cancer cells which could cause paresthesia and/or deviation of the tongue clinically in patients with TSCC. In contrast, up to now, the presence and role of nerves in the tumor microenvironment of TSCC have received little attention. Recently few studies have revealed a converse phenomenon that nerve fibers can sprout into the tumor microenvironment initiatively, termed "tumor neoneurogenesis", and a new paradigm has been raised that the newly growing nerves plays an important role in cancer development and dissemination [4-6]. The innervation of the tongue is complex, a further identification of tumor neoneurogenesis in TSCC may provide a new insight into its pathogenesis and lead to a novel anti-cancer strategy.

A pilot research showed that neoneurogenesis was observed in TSCC tumor microenvironment and might play an important role in its pathogenesis [7]. However, the relationship between neoneurogenesis and development of TSCC has not been drawn much attention, a study was designed to investigate whether neoneurogenesis is a risk factor to the progression and cervical lymph node metastasis of TSCC.

\section{Methods}

\section{Research groups}

Twenty patients with TSCC were selected from inpatient medical record database of Tianjin Stomatological Hospital of Nankai University from May 2019 to August 2019. All the cases were undertaken locally extended resection combined with radical neck dissection simultaneously. The specimens of primary tumors and lymph nodes were gained and fixed in $4 \%$ paraformaldehyde, embedded in paraffin, sectioned respectively. The pathological type of primary tumor and lymph node metastasis were re-confirmed by the pathologist. The study was approved by the Tianjin Stomatological Hospital Ethics Committee, and informed consent written was 
obtained from all participants. All the cases were divided into 4 groups according to WHO T-stage standard (five persons in each group) [8]. In the same way, the objectives were divided into 2 groups on the basis of cervical lymph node metastasis or free.

\section{Immunohistochemistry histological Studies}

Paraffin sections from primary tongue cancer tissues were stained using routine immunohistochemistry method with a monoclonal anti-Neurofilament Light chain antibody (NF-L antibody is purchased from Novus Technology: NB300-132). Positive staining of NF-L was considered as having new growth of nerve fibers (namely neoneurogenesis). The number of newly formed nerve bundles was calculated in each randomized view from 3 nonoverlapping microscope fields of each tumor section under a light microscope at X 200 .

\section{Statistical analysis}

The analyses were performed using SPSS 22.0 software. Results were expressed as Mean \pm SD. The relative difference in new growth fiber bundles density among T1-T4 groups were assessed using the Tukey range test post hoc t-tests for multiple comparisons. When there were only two groups in lymph node metastasis or not, a standard t-test was used to analyze the data. Chi-square test was performed to calculate the $P$ value. An $\otimes$ level of 0.05 was considered statistically significant.

\section{Results}

\section{Characteristics of patients}

The age of patients with malignancies varied from 33 to 75 years with a mean age of $56.10 \pm 11.18$ years. Males were affected predominantly with a ratio of $1.5: 1$ to females (12:8) (Shown in Table-1).

\section{IHC staining in primary tumor}

IHC staining of NF-L in paraffin sections of TSCC was positive in all 20 cases, which suggested that the newly formed nerves could infiltrate into the tumor mciroenvironment (namely neoneurogenesis were observed).

\section{Neoneurogenesis in different T-stages of TSCC}

According to WHO T-stage standard, patients with TSCC were divided into 4 groups: T1DT2ロT3 and T4.

Prevalence of intensity of newly formed nerve fiber bundles increased with the aggression of T-stages, and the difference was statistically significant between early stage (T10T2) and late stage (Т3०Т4) ( $p=0.000$, shown in Figue-1 and Table-2).

\section{Neoneurogenesis in cervical lymph node metastasis of TSCC}

Fifty percent of objectives in our study were affected by lymph node metastasis (Shown in Table-1). Two groups were gained on the basis of lymph node metastasis ( N1) or not (N0) in patients with TSCC.

Increased intensity of newly growth nerve fiber bundles was observed in patients with lymph node metastasis, and the difference was statistically significant $(p=0.000$, shown in Figue- 2 and Table- 3$)$.

\section{Discussion}

One of the most well known hallmarks of cancer cells is their ability to break away from their original site to invade neighboring tissue and spread to distant body parts [9].

Cancer is a complex and heterogeneous disease involving the interaction between cancer cells and the stroma冈including endothelial cells, immune cells, fibroblasts, and the extracellular matrix $\bigotimes$ to create diverse tumor microenvironments, which can facilitate cancer progression and dissemination and lead to innovative anticancer therapies, for example, those targeting angiogenesis [2,10].

In the research domain of tumor microenvironment, the role of tumor angiogenesis and lymphogenesis in tumorigenesis and metastasis has been well demonstrated. As an important component of tumor microenvironment, despite early studies have shown a very close relationship between nerves and malignant tumors, such as local sensory disorders (numbness, pain) and motor disorders 
(hemiplegia, facial paralysis) when nerves were invaded by cancer cells, however, the role of nerves in tumorigenesis and development has received relatively little research attention compared to angiogenesis and lymphogenesis $[11,12]$.

Previously, when concerning about the relationship between nerves and tumor cells, nerves had been recognized to be passively affected by cancer cells in the process of perineural invasion (PNI) $[13,14]$. Until recently, in a subverted breakthrough article, Magnon has observed a distinctive phenomenon that nerves can infiltrate the tumor microenvironment proactively by new sprouted growing fibers (namely tumor neoneurogenesis) for the first time, and provided evidence that autonomic nerves contributed to prostate cancer pathogenesis and development in the mouse model [4]. The identification of neoneurogenesis in tumor microenvironment provides a new perspective in its oncogenesis research and may offer a potential value of anti-neurogenic therapeutic treatment in cancer [10,1517].

Neurofilaments (NF) are the 10 nanometer or intermediate filaments found in neurons. They are a major component of the neuronal cytoskeleton, and are believed to function primarily to provide structural support for the axon and to regulate axon diameter.

Three major neurofilament subunits are divided based upon the apparent molecular mass of the mammalian subunits on SDSPAGE:the light (NF-L) runs at 68-70 kDa, the medium (NF-M) runs at about 145-160 kDa and the heavy (NF-H) runs at 200-220 kDa [18].

NF-L and NF-H are confirmed to mark newly formed and mature nerve fibers, respectively [19].

Numb or local pain and impoverished mobility of the tongue are among the most common presentations of tongue cancers when linguial and hypoglossal nerves affected, especially in the late stage of TSCC. In traditional views between the relationship of tongue cancer cells and nerves, nerve fibers are passively affected by cancer cells in tumor microenvironment via PNI [20].

Recently Xu discovered that IHC staining of NF-L was positive in 10 paraffin sections of TSCC in their study which suggested that the tongue cancer recruited newly formed nerves in its microenvironment; in an animal model, IHC staining of NF-L was positive even in the initial phase of tumor formation and the number of newly formed nerve fibers increased accompanied with growth of the tumors indicating that neoneurogenesis might attribute to the pathogenesis of TSCC [7].

In our studies, neoneurogenesis in TSCC was confirmed by positive staining of NF-L suggesting that new growth nerves had been observed in tumor tissues, namely the newly formed nerve fibers can infiltrate tumor microenvironment proactively. Intensity of newly formed nerve fiber bundles increased with the progression of T-stages, and the difference was statistically significant between early stage ( T1 1 T2 ) and late stage ( T3ロT4 ). In patients with lymph node metastasis, increased intensity of newly growth nerve bundles was observed, and the difference was statistically significant to those without metastasis. The results above indicated that neoneurogenesis was a predisposing factor to the progression and metastasis of TSCC.

The exact linkage between neoneurogenesis and tumorigenesis of TSCC has not been uncovered, the underlying mechanisms may

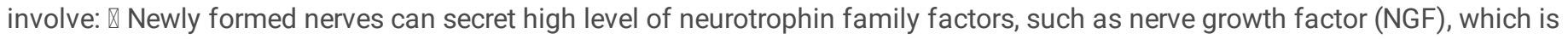

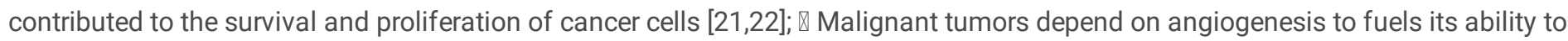
grow and spread, endothelial $\beta$-adrenergic receptor signaling via adrenergic nerve-derived noradrenaline is critical for activation of an angiogenic switch to promote tumor angiogenesis which can facilitate rapid tumor growth [23-25]; $\varangle \quad \beta 2$-adrenergic receptor ( $\beta 2$-AR) has been reported to be a potential promoter to cancer initiation and aggressiveness in various types of solid cancer [26]. Liu discovered that increased expression of $\beta 2$-AR was correlated with differentiation, lymph node metastasis and reduced overall survival rate in patients with TSCC [27]. While $\beta 2$-AR activation has a positive influence on nerve regeneration and has been found to enhance neurogenesis [28]囚thus up-regulation of $\beta 2$-AR signaling in the process of neoneurogenesis could result in cell proliferation and promote tumor metastasis in TSCC .

Surgery is still the most important treatment in TSCC. Tongue cancer canbe highly curable if detected early, however, tissue defects created by extensive resection can severely affect speech and swallowing function as well as destroy the aesthetic appearance at late stage. Despite current advances in its diagnosis and management, the tongue cancer remains a difficult region to assess and a 5-year survival remains poor at $62.7 \%$ in the USA and below $50 \%$ in other countries due to local recurrence and distant metastatic spread $[29,30]$. Thus, further study into its pathogenesis and predictors of outcome is critical to devise a new preventive measure, clarify an earlier diagnosis, and offer a novel treatment modality.

Neoneurogenesis has been proved as a risk factor to the aggravation and cervical lymph metastasis of tongue cancer in our research, thus, denervation should be emphasized in making a treatment plan by oral and maxillofacial surgeons. Deep research in the cross-talk

Page 4/8 
between neo-nerves and tongue cancer cells could provide a new insight into its basic mechanism of oncogenesis and develop a possibility of anti-neurogenesis therapy in the future.

\section{Conclusion}

Neoneurogenesis in tumor microenviroment of TSCC is a predisposing factor to its aggressive development and a promoter to the metastasis of cervical lymph node. From a therapeutic perspective, further studies on this topic may provide a new clinical opportunity in its management through anti-neurogenesis approach.

\section{Abbreviations}

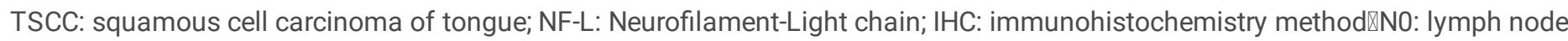
metastasis free; N1: lymph node metastasis.

\section{Declarations}

Ethics approval and consent to participate: The study was approved by the Tianjin Stomatological Hospital Ethics Committee,and informed consent written was obtained from all participants.

Consent for Publication: Not applicable

Availability of data and material: We declared that materials described in the manuscript, including all relevant raw data, will be freely available to any scientist wishing to use them for non-commercial purposes, without breaching participant confidentiality.

Competing interests: The authors declare that they have no competing interests.

Funding: Not applicable

Authors' contributions:

ZX, designs the study and writes the paper;

JS, doses the experiment of $\mathrm{IHC}$ and collects data;

$X X, L L, Y Y$ and JZ, provide the sample of tumor tissues.

Acknowledgements: Not applicable

\section{References}

[1] Ren ZH, Wu HJ, Zhang S, et al.

A new surgical strategy for treatment of tongue squamous cell carcinoma based on anatomicstudy with preliminary clinical evaluation. J Craniomaxillofac Surg. 2015;43(8):1577-82.

[2] Hui L, Chen Y. Tumor microenvironment: Sanctuary of the devil. Cancer Lett. 2015;368(1):7-13.

[3] Alaeddini M, Etemad-Moghadam S. et al. Lymphangiogenesis and angiogenesis in oral cavity and lower lip squamous cell carcinoma. Braz J Otorhinolaryngol. 2016;82(4):385-90.

[4] Magnon C, Hall SJ, Lin J, et al. Autonomic nerve development contributes to prostate cancer progression. Science. 2013;341(6142):1236361.

[5] Zhao Q, Yang Y, Liang X, et al. The clinicopathological significance of neurogenesis in breast cancer. BMC Cancer. 2014;14:484.

[6] Cole SW, Nagaraja AS, Lutgendorf SK, et al. Sympathetic nervous system regulation of the tumour microenvironment. Nat Rev Cancer. 2015;15(9): 563-72. 
[7] Xu Zhq, Shang JW. Neoneurogenesis in Squamous Cell Carcinoma of Tongue: A New Mechanism for Its Development. 0 J Stoma. 2019;9(5):102-7.

[8] Huang SH, O'Sullivan B. Overview of the 8th Edition TNM Classification for Head and Neck Cancer. Curr Treat Options Oncol. 2017;18:40.

[9] Hanahan D, Weinberg RA. Hallmarks of cancer: the next generation. Cell. 2011;144(5):646-74.

[10] Jobling P, Pundavela J, Oliveira SM, et al. Nerve-Cancer Cell Cross-talk: A Novel Promoter of Tumor Progression. Cancer Res.2015;75(9):1777-1781.

[11] Roh J, Muelleman T, Tawfik O, et al. Perineural growth in head and neck squamous cell carcinoma: a review. Oral Oncol. 2015;51(1):16-23.

[12] Ferrari F, Forte S, Ardighieri L, et al. Multivariate analysis of prognostic factors in primary squamous cell vulvar cancer: The role of perineural invasion in recurrence and survival. Eur J Surg Oncol. 2019;45(11):2115-9.

[13] Liebig C, Ayala G, Wilks JA. Perineural invasion in cancer: a review of the literature. Cancer. 2009;15(15):3379-91.

[14] Mancino M, Ametller E, Gascón P, et al. The neuronal influence on tumor progression. Biochim Biophys Acta. 2011;1816(2):105-18.

[15] Zhao CM, Hayakawa Y, Kodama Y, et al. Denervation suppresses gastric tumorigenesis

. Sci Transl Med. 2014;6(250):250ra115.

[16] Lu R, Fan C, Shangguan W, et al. Neurons generated from carcinoma stem cells support cancer progression. Signal Transduct Target Ther. 2017;2:16036.

[17] Faulkner S, Jobling P, March B, et al. Tumor Neurobiology and the War of Nerves in Cancer. Cancer Discov. 2019;9(6):702-10.

[18] Löhrke S, Brandstätter JH, Boycott BB, et al. Expression of neurofilament proteins by horizontal cells in the rabbit retina varies with retinal location. J neurocytol. 1995;24 (4):283-300.

[19] Carden MJ, Trojanowski JQ, Schlaepfer WW, et al. Two-stage expression of neurofilament polypeptides during rat neurogenesis with early establishment of adult

phosphorylation patterns. J Neurosci. 1987;7(11):3489-504.

[20] Tarsitano A, Tardio ML, Marchetti C. Impact of perineural invasion as independent prognostic factor for local and regional failure in oral squamous cell carcinoma. Oral Surg Oral Med Oral Pathol Oral Radiol. 2015;119(2):221-8.

[21] Shen WR, Wang YP, Chang JY, et al. Perineural invasion and expression of nerve growth factor can predict the progression and prognosis of oral tongue squamous cell carcinoma. J Oral Pathol Med. 2014;43(4):258-64.

[22] Griffin N, Faulkner S, Jobling P, et al. Targeting neurotrophin signaling in cancer: The renaissance. Pharmacol Res. 2018;135:12-7.

[23] Hayakawa Y, Wang TC. Nerves switch on angiogenic metabolism. Science. 2017;358(6361):305-6.

[24] Zahalka AH, Arnal-Estapé A, Maryanovich M, et al. Adrenergic nerves activate an angio-metabolic switch in prostate cancer. Science. 2017;358(6361):321-6.

[25] Hondermarck H, Jobling P. The Sympathetic Nervous System Drives Tumor Angiogenesis. Trends Cancer. 2018;4(2):93-4.

[26] Cole SW, Sood AK. Molecular pathways: beta-adrenergic signaling in cancer. Clin Cancer Res. 2012;18(5):1201-6.

[27] Liu H, Wang C, Xie N, et al. Activation of adrenergic receptor $\beta 2$ promotes tumor progression and epithelial mesenchymal transition in tongue squamous cell carcinoma. Int J Mol Med. 2018;41(1):147-54. 
[28] Chai GS, Wang YY, Yasheng A, et al. Beta 2-adrenergic receptor activation enhances neurogenesis in Alzheimer's disease mice. Neural Regen Res. 2016; 11(10): 1617-24.

[29] Prince S, Bailey BM. Squamous carcinoma of the tongue: review. Br J Oral Maxillofac Surg. 1999;37(3):164-74.

[30] Kolokythas A, Park S, Schlieve T, et al. Squamous cell carcinoma of

the oral tongue: histopathological parameters associated with outcome. Int J Oral Maxillofac Surg. 2015;44(9):1069-74.

\section{Tables}

Due to technical limitations the tables are available as a download in the Supplementary Files.

\section{Figures}

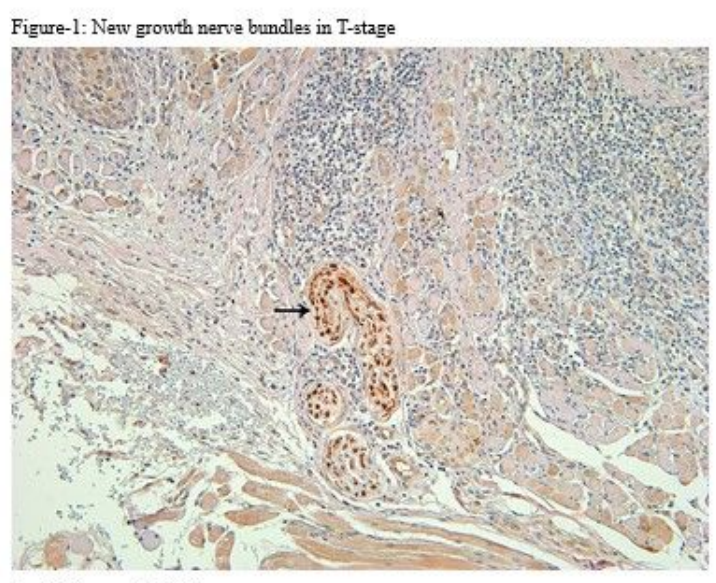

la: $\mathrm{Tl}$ stage $(\mathrm{X} 200)$

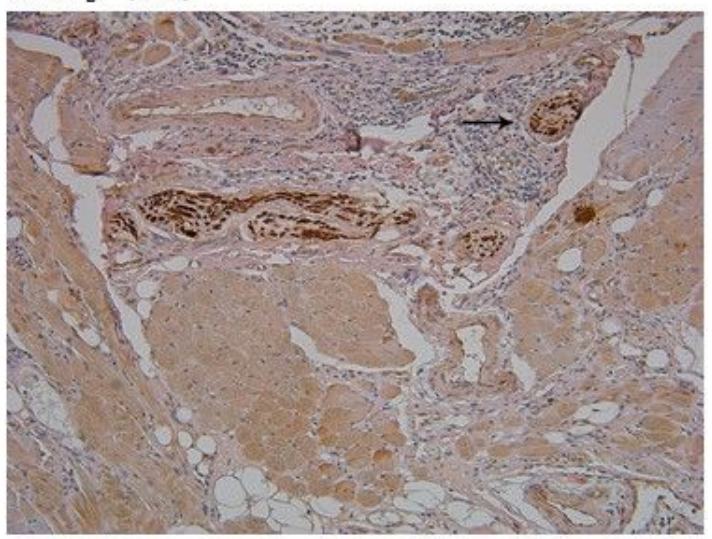

lb: T2 stage $\left(X_{2} 200\right)$

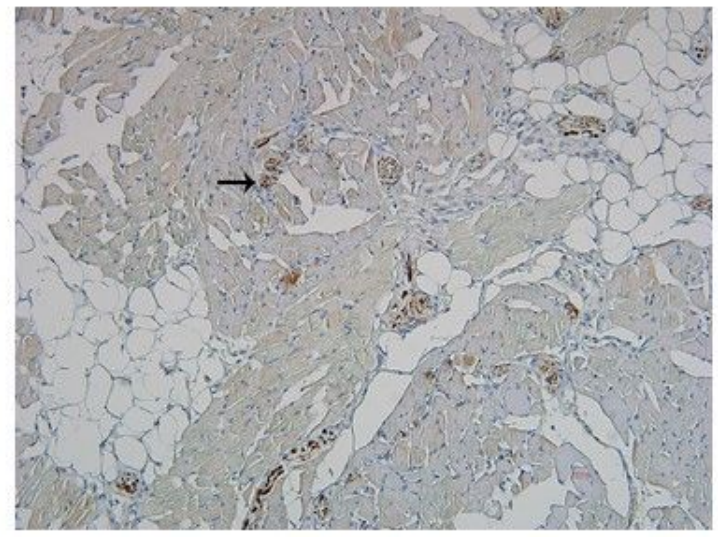

lc: T3 stage (X200)

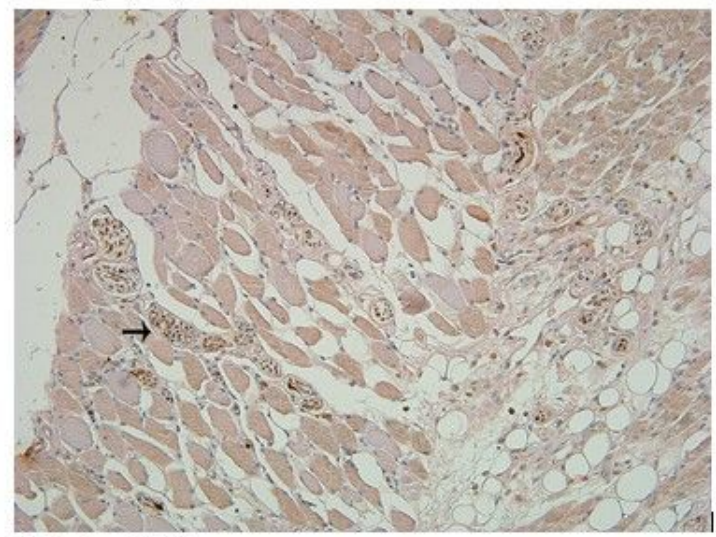

1d: T4 stage (X200)

\section{Figure 1}

New growth nerve bundles in T-stage 


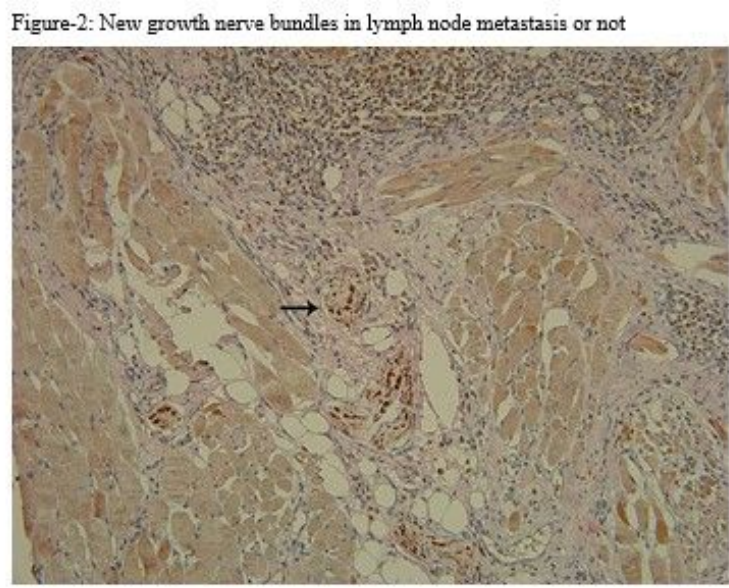

2a: No $(\mathrm{X} 200)$

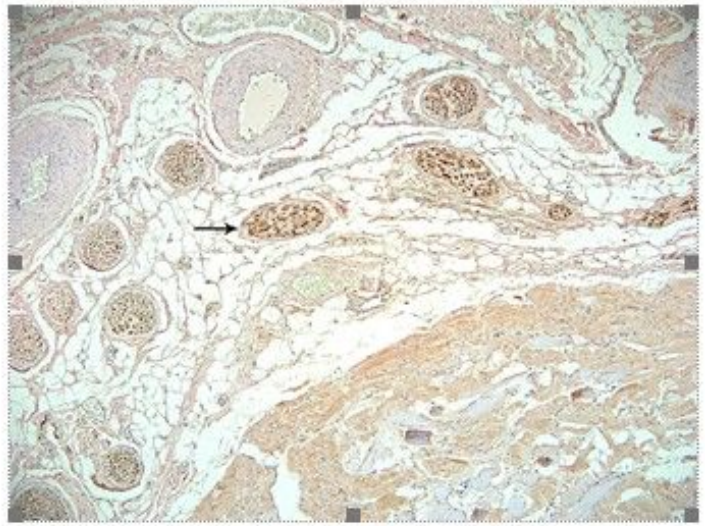

2b: N1 (X200)

(N0: lymph node metastasis free, $\mathrm{Nl}$ : lymph node metastasis, ${ }^{*} \mathrm{p}<0.01$ )

\section{Figure 2}

New growth nerve bundles in lymph node metastasis or not

\section{Supplementary Files}

This is a list of supplementary files associated with this preprint. Click to download.

- Tablesrevised.pdf 